\title{
Qualitative process study of community pharmacist brief alcohol intervention effectiveness trial: can research participation effects explain a null finding?
}

Article

Accepted Version

Creative Commons: Attribution-Noncommercial-No Derivative Works 4.0

Quirk, A., MacNeil, V., Dhital, R., Whittlesea, C., Norman, I. and McCambridge, J. (2016) Qualitative process study of community pharmacist brief alcohol intervention effectiveness trial: can research participation effects explain a null finding? Drug and Alcohol Dependence, 161. pp. 36-41. ISSN 03768716 doi: https://doi.org/10.1016/j.drugalcdep.2016.01.023 Available at https://centaur.reading.ac.uk/80758/

It is advisable to refer to the publisher's version if you intend to cite from the work. See Guidance on citing.

To link to this article DOI: http://dx.doi.org/10.1016/j.drugalcdep.2016.01.023

Publisher: Elsevier

All outputs in CentAUR are protected by Intellectual Property Rights law, including copyright law. Copyright and IPR is retained by the creators or other copyright holders. Terms and conditions for use of this material are defined in the End User Agreement. 


\section{www.reading.ac.uk/centaur}

\section{CentAUR}

Central Archive at the University of Reading

Reading's research outputs online 


\title{
open OAccess
}

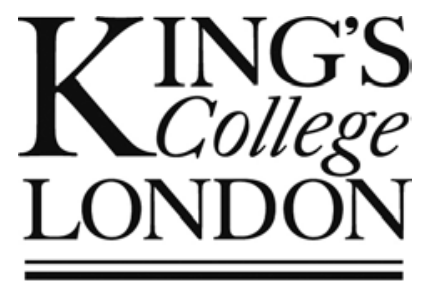

\section{King's Research Portal}

\author{
DOI: \\ 10.1016/j.drugalcdep.2016.01.023 \\ 10.1016/j.drugalcdep.2016.01.023
}

Document Version

Peer reviewed version

Link to publication record in King's Research Portal

Citation for published version (APA):

Quirk, A., MacNeil, V., Dhital, R., Whittlesea, C., Norman, I., \& McCambridge, J. (2016). Qualitative process study of community pharmacist brief alcohol intervention effectiveness trial: Can research participation effects explain a null finding? DOI: 10.1016/j.drugalcdep.2016.01.023, 10.1016/j.drugalcdep.2016.01.023

\section{Citing this paper}

Please note that where the full-text provided on King's Research Portal is the Author Accepted Manuscript or Post-Print version this may differ from the final Published version. If citing, it is advised that you check and use the publisher's definitive version for pagination, volume/issue, and date of publication details. And where the final published version is provided on the Research Portal, if citing you are again advised to check the publisher's website for any subsequent corrections.

\section{General rights}

Copyright and moral rights for the publications made accessible in the Research Portal are retained by the authors and/or other copyright owners and it is a condition of accessing publications that users recognize and abide by the legal requirements associated with these rights.

- Users may download and print one copy of any publication from the Research Portal for the purpose of private study or research. - You may not further distribute the material or use it for any profit-making activity or commercial gain

- You may freely distribute the URL identifying the publication in the Research Portal

\section{Take down policy}

If you believe that this document breaches copyright please contact librarypure@kcl.ac.uk providing details, and we will remove access to the work immediately and investigate your claim. 
$\underline{\text { Title }}$

Qualitative process study of community pharmacist brief alcohol intervention effectiveness trial: can research participation effects explain a null finding?

Authors

Alan Quirk ${ }^{a},{ }^{*}$, Virginia MacNeil ${ }^{b}$, Ranjita Dhital ${ }^{c}$, Cate Whittlesea ${ }^{d}$, Ian Norman ${ }^{\mathrm{e}}$, Jim McCambridge ${ }^{\mathrm{b}, \mathrm{f}}$

a. Royal College of Psychiatrists' Centre for Quality Improvement, London, UK

b. Department of Social and Environmental Health Research, London School of Hygiene and Tropical Medicine, London, UK

c. National Addiction Centre, Institute of Psychiatry, Kings College London, UK

d. School of Medicine, Pharmacy and Health, Durham University, UK

e. Florence Nightingale School of Nursing and Midwifery, Kings College London, UK

f. Department of Health Sciences, University of York, UK

*Corresponding author. Tel. +44 203701 2699. E-mail address: aquirk@rcpsych.ac.uk (A. Quirk) 


\section{ABSTRACT}

\section{Aims}

This qualitative process study, nested within a randomised controlled trial evaluating community pharmacist brief alcohol intervention delivery, aims to explore participants' engagement with the trial, so as to identify whether research participation effects may explain why the brief intervention was not found to be effective.

\section{Method}

Qualitative interviews were conducted with 24 randomly selected participants approximately one month after the end of the trial. Semi structured Interviews were conducted by telephone in which participants were asked to give a chronological account of their trial participation, leading to a discussion of possible impacts. These were digitally recorded, transcribed verbatim, and analysed using the Framework method.

\section{Results}

A range of motivations for taking part in the trial were identified, including pharmacy visits wanting to obtain an assessment of their drinking. Participants in both arms of the trial spoke of the potent effect that screening had on them. All participants were exposed to discussions about alcohol with empathic pharmacists, and as this is an integral intervention component, this constitutes contamination. Participants' pre-existing ideas about the nature of alcohol problems had an important bearing on how relevant they thought the intervention was to them.

\section{Conclusion}

A detailed appreciation of participant engagement with the trial can provide a strong basis for interpretation of trial outcome data, and in this instance helps explain the null finding. Other findings indicate the need for dedicated studies of public understanding of the nature of alcohol problems, and their implications for receptivity to brief interventions. 


\section{INTRODUCTION}

There has been limited previous study of the experiences of participants involved in randomised controlled trials evaluating behavioural interventions. Qualitative studies have helped to illuminate the complexities involved in trial recruitment and retention, with, for example, participants' prior and continuing convictions about its purpose, nature and conduct all highly relevant (Scott et al., 2011). Process studies are now accepted as offering valuable data on study and intervention implementation issues in trials, and potentially into how interventions exert their effects or are not effective (Oakley et al., 2006). Systematic reviews and hypothesis generating narrative reviews have investigated the possibility that taking part in these types of studies, and indeed taking part in research more generally, may impact upon participants in ways which are routinely ignored (McCambridge et al., 2011; McCambridge et al., 2014a, 2014b, 2014c). Any such "research participation effects" may be important if they impact on the data that research studies gather, introducing bias and obstructing valid inference generation. These concerns have been prominent in alcohol research (Jenkins et al., 2009). For around 40 years there have been observations that having drinking and related problems assessed by interview or by completing questionnaires, may itself alter research participants' thinking about their drinking (Gallen, 1974; Sobell and Sobell, 1981; Obbourne and Annis, 1988). This may occur when people take stock of their situation and think differently about, or decide to change, their behaviour (Clifford and Maisto, 2000). This possibility is congruent with a self-regulation perspective on motivation and behaviour and implies some degree of bias in conventionally undertaken research studies in which participant drinking is routinely assessed (McCambridge, 2009).

Brief interventions are relatively simple means of helping people to consider their drinking, offered widely within the population (Heather, 1989). They usually take the forms of face-to-face conversations with health professionals or automated internet interventions (McCambridge, 2011). Although there is limited understanding of both their detailed content, and exactly how they may exert any effects (McCambridge, 2013; Gaume et al., 2014), it is quite likely that they do so by similar means to those described for the effects of being assessed. Almost all randomised controlled trials evaluating brief interventions assess all their participants in the course of evaluation study. If assessment does alter behaviour, then these trials will be biased by any interaction of assessment and brief intervention (McCambridge et al., 2011; McCambridge et al., 2014a, 2014b). Systematic review data indicate small effects of assessments on behaviour in randomised comparisons within brief alcohol intervention trials, with the likely implication that these studies under-estimate the true effects of these interventions (McCambridge and Kypri, 2011). There may also be other aspects of taking part in these studies that engender thinking about drinking, and hence confound assessment and wider research participation effects with those of the interventions being evaluated. 
There has been one previous qualitative study of research participation effects (McCambridge et al., 2014d). This weight loss trial process study found that reasons for participation including allocation preferences exerted far reaching influence on how participants engaged with the trial procedures. The aim of the present study was to use qualitative data from a process study nested within a community pharmacist brief intervention trial, the first as far as we are aware in this field of study, to study research participation effects. When we decided to do this study we were unaware of the trial's null findings; both groups reported modest reductions in drinking, with outcomes similar to each other (Dhital et al., 2015). These data allow us to examine whether research participation effects may explain the trial's findings.

\section{METHODS}

\section{Parent trial}

This paper draws on a qualitative study of participant experiences of a randomised controlled trial designed to determine if brief alcohol interventions delivered by community pharmacists were effective in reducing hazardous and harmful drinking amongst pharmacy customers after three months in comparison with a leaflet only control condition. The trial protocol is reported elsewhere (Dhital et al., 2013). This was a highly naturalistic study with approximately half of all pharmacists in a London borough participating, all having a consultation room available for interventions delivery. The pharmacists received seven hours training on trial procedures and intervention delivery, involving communication skills training influenced by the perspective of motivational interviewing (Miller and Rollnick, 2012). This emphasises listening with empathy, the importance of the careful use of language in a coaching style, and helping people to make their own decisions by avoiding direct persuasion. Pharmacy support staff working with recruited pharmacists attended a brief training session on how to identify potentially suitable participants for the trial.

Pharmacists and support staff introduced the study to pharmacy visitors. They asked a single question about drinking ("How often do you have three or more drinks on a single occasion?") and provided study information. Customers who reported consuming three or more drinks monthly or more regularly were invited to have their alcohol use assessed further with the Alcohol Use Disorders Identification Test (AUDIT, (Babor et al., 2001)) questions asked by the pharmacist in the consultation room. Participants who fulfilled the study criteria (aged 18 years or older and hazardous or harmful drinkers, excluding drinkers with likely dependence i.e. with AUDIT scores 8-19 inclusive) and were willing to participate gave formal consent. Participants were then randomised in equal numbers to either brief intervention or leaflet-only control condition. Pharmacists were blind to 
allocation status until the point at which they opened the sealed envelopes. Thereafter they were not involved in research data collection. For the purposes of both follow-up study and data management, relevant personnel were blinded to randomisation status throughout the trial (Dhital et al., 2013).

The purpose of the brief intervention was to encourage participants to think further about their drinking and whether they should reduce it, and if they are ready to do so, to discuss how. Participants allocated to the intervention condition were offered a discussion of approximately 10 minutes duration. This intervention contains a number of structured components in the form of an intervention protocol (Dhital et al., 2013). The conversation began by building rapport through asking questions about participants' experience of answering the AUDIT screening questions. Participants were then encouraged to talk about how drinking fitted in with their lives, explore any ambivalence and elicit their evaluation of their drinking, including any problems associated with it. The conversation closed by either the participant or the pharmacist providing a summary of the conversation. The intervention and control groups were given different sets of information (discussed further below).

\section{Qualitative process study}

After three months, trial outcome data collection involved a telephone interview. At this point, participants were asked if they were interested in participating in a further telephone call to explore experiences of participating in the trial (Dhital et al., 2013). Formal consent for the process study was separately obtained alongside trial recruitment by the pharmacist. Of all those who gave consent (326/407 trial participants), 291 were followed-up for the trial. From this pool, 24 participants, stratified by study condition (12 from each), were randomly selected to participate in the process study; all agreed to participate when called. Characteristics of the interviewed sample are given in Table 1. These participants were contacted approximately one month after the threemonth trial follow-up telephone call, for a 20 minute discussion by telephone with RD. Study resources were limited so telephone interviewing was chosen over face-to-face interviewing for the benefits of cost effectiveness and time efficiency (Sarantakos, 1998). The separation between interviewer and interviewee can present challenges for interpersonal communication, specifically in the formation of trust (Groves \& Kahn, 1979), and with interviewees typically providing relatively less detail and elaboration than in face to face interviewing (Irvine, 2011). It is therefore appropriateto acknowledge the limited depth of understanding that could be expected in these short telephone interviews, which we were aware of at the outset. A semi-structured topic guide (see Appendix) was developed for this study. This provided the basis for a chronological account of study participation including interventions delivery, leading to a discussion of possible impacts of 
trial participation. Those who completed the telephone interview were sent a $f 10$ gift voucher as a token of appreciation for participation.

\section{[Insert Table 1]}

The telephone discussion was digitally recorded and professionally transcribed verbatim. Transcripts were imported into a qualitative data analysis programme (NVivo10). The highly structured nature of the qualitative data lent itself to Framework Analysis, which was used to systematically code and analyse the data, using a matrix to summarize and compare the transcripts by participant and theme (Ritchie and Spencer, 2002). The value of this approach is that the data are ordered into descriptive 'chunks' which aids theme focused analysis (the analyst looks down the columns to examine all interviewees' responses in relation to a particular issue), while the matrix keeps the context of the data and aids the search for explanation (by looking across rows). The themes were partly drawn from the topic guide and later refined to include emerging and unexpected material in the data. VM initially identified and coded the data which was then reviewed by $A Q$ and JM and the same three authors discussed and agreed how the data should be interpreted. AQ led the writing of the results after the first draft prepared by VM. The notation used below for individual participants identifies a unique number preceded by ' $I$ ' and ' $C$ ' for intervention and control participants respectively.

\section{RESULTS}

\section{Recruitment to the trial and reasons for participation}

A quarter of the people we interviewed said that they had taken part because they wanted to find out "where [they] stand" as a drinker:

...I thought it was a good idea. Well, it's for health reasons as well and I think it tells you if you're a very heavy drinker or a light drinker. $\mathrm{C} 01$

I thought it could be quite interesting for me to know where I stand. I12

I wanted to find out a bit more about what the alcohol study was about, whether it was going to moderate my drinking, or how much I was drinking was affecting my health and my emotional well-being, if I'm being honest. 124

A few interviewees gave just one single reason for participating in the trial but more identified a range of factors as having influenced their decision. Two-thirds cited altruism:

it's good to take part in these sort of things because I mean I'm not saying it wasn't beneficial to me, don't get me wrong, but if you don't help with these sort of things then you're not going to help find a process or get a cure or help people if you don't help the research. 113 
A recurrent theme was the importance of a trusting, pre-existing relationship between participant and pharmacist. The perceived familiarity of the community pharmacist, suggest there are parallels with the doctor/patient model in this regard:

He's a very nice chap in there, he's looked after my father over the years and l've come to know him quite well. 121

In addition, pharmacists' friendly manner, and the perception that it was a place where "you probably wouldn't feel judged", contributed to pharmacy customers agreeing to take part:

The pharmacist who served me told me about the study and was very friendly in the way that she did so, which definitely encouraged me. I14

It's a place where you probably wouldn't feel judged. 123

\section{Screening/assessment}

Customers who reported consuming three or more drinks monthly or more regularly were invited to have their alcohol use assessed further using the AUDIT in the second stage of screening by the pharmacist in the consultation room. Once the participants had been given their AUDIT scores, they were advised that they may be drinking more than is recommended (Babor et al., 2001) and were eligible for participation in the study. After consent being obtained they were randomised in equal numbers to either the brief intervention, which was delivered immediately afterwards, or the information leaflet-only control condition. The process of being assessed and fed back the results reportedly had little effect on about half of all participants, some of whom invoked ideas about problem drinking:

I don't feel that I've actually got a problem with alcohol, that I drink excessively. I13

I know a lot of heavy drinkers, in the building game there is a lot of heavy drinkers, and maybe I was one a few years ago, but I've never got up in the morning and been dependent on a drink, even when I was drinking heavily. C07

However, other participants spoke of being affected by assessment, sometimes profoundly, in one of two ways. First, simply responding to questions about their drinking and the impact it has on their lives, could be surprising in that it made participants aware they were drinking "more than I realised":

Some of the questions that were put before me, I was quite shocked in some of my own replies. 113 
I probably drink more than I realised, it's just that you don't think about it until someone asks you to number something and you think God, actually I probably drink two bottles of wine on the weekend. 123

Second, it was being advised that their drinking was unhealthy or excessive that was "pretty scary" for this participant with an AUDIT score of 19:

She said that I was close to the mark. I think I was one point away from where she would have had to refer me to a GP for alcohol treatment. So that was pretty scary. I16

In contrast, others felt reassured by the communication of their eligibility because they thought their drinking would have been classified as "much worse than that" and it made had them realise it was actually "not that much":

On the whole I was quite shocked at my result. It was quite good. I thought it would be worse than that $\mathrm{CO} 3$

It made me realise that I don't drink so much, so I did feel better about myself..........because the way the questions were asked made me think about when I drink, and how frequently I drink, and made me realise that it's not that much. $\mathrm{CO} 2$

The AUDIT identifies risky but not necessarily problematic drinking and the pharmacists had been trained to feed back the results in a dispassionate and non-judgemental way. But this did not always happen, indicating some implementation failure. Several participants reported that the pharmacist had been at pains to reassure them that their drinking was not excessive, thus departing from the study protocol:

I thought I was excess. And when he explained to me, he said, no, you're not excess, you're OK on your drinking wise. He said, your health shouldn't suffer that much. And I thought that was good. C01

One participant evidently misunderstood his situation, which may have been because it had not been communicated clearly by the pharmacist:

I wasn't told that I was drinking more than the recommended amount because I don't. I'm not a huge drinker though. $\mathrm{CO}$

\section{The Brief Intervention}

All 12 intervention participants we interviewed said that their pharmacist had been understanding or empathic, as they were meant to have been with this group:

I didn't feel like I was under the spotlight, it was, more a relaxed conversation, like what I'm having with you now. It just didn't feel like any pressure to me, anyway, as I say I've not got a problem. Someone with a problem might not want to talk about it, I don't 
know, denial and all that malarkey. But I felt quite at ease and quite happy to speak to him. 113.

The limited effects of the intervention are suggested by the absence of risk or problem identification in the quotation above. This participant, however, went on to articulate something close to the intended prevention effects for those who do not have alcohol problems (the intended effects for those who do have current problems would be to help reduce them):

When we started to get into the conversation and taking part and, it sort of opened my eyes to, I'm not a weekly drinker, I'm not an excessive drinker, I don't binge drink, but there was a few little things that came to light that are not a problem. But there's times when I could have sort of not drunk but I did drink, if you know what I mean. It's just a little bit of an eye opener really. 113

\section{Printed information}

After the ten minute discussion, the intervention group was given the "Units and You" booklet, a "Unit/Calorie Calculator Wheel" and an alcohol services leaflet to take away. This additional intervention component was valued, especially the information about unit recommendations and calorific information:

The best thing that she gave me was the unit and calorie counter, which I still have actually on my pin board because it's very, very interesting. I was sort of on a mission to, as I continue to be, to lose some weight. So if anything, that was very beneficial to provide for me. 122

Another participant thought that the discussion (BI) was inappropriately targeted at her and that she found the printed material more useful:

It was more the wheel, there was a leaflet as well, rather than the conversation. I think the conversation was probably more directed at someone who maybe had experienced issues of severe, heavy drinking and things or other social issues around it. 119

Some participants said they still looked at it from time to time because the information was very useful while another said he had not read any of the material as he preferred the discussion with the pharmacist.

Participants allocated to the control condition were not explicitly informed that they were control participants and were given a leaflet entitled "Alcohol: The Basics"., the content of which was not expected to be effective at promoting behaviour change. Again there were protocol departures:

I didn't read it all because he also gave a talk about it, the units and everything else so really for what I read is what he was explaining to me. I wouldn't say I sat down and read it indoors because he was explaining everything for you. $\mathrm{C01}$ 
Others said they found the information useful and that it had had an impact on their thinking and behaviour:

The leaflet made me think about things .........and in this case thinking about my drinking meant I drank slightly less. C05

The pharmacists undertook a half-day training course on skilful listening and communication skills in preparation for brief intervention delivery in the trial. However approximately half of the information leaflet-only control participants commented on the pharmacists' professional, calm and understanding manner, which suggests that the pharmacists were using similar empathic communication skills with both groups. In trials terms, this is contamination, with the control group being exposed to an integral component of the intervention being evaluated.

\section{Perceived impact of participation}

We asked both groups about the impact that taking part in the study had had on them. The aim of the brief intervention was to promote self-evaluation, facilitated by the pharmacist, but about half of the intervention group said that taking part had not changed their thinking or their drinking, because they did not perceive themselves to have a problem anyway. Others said that it had "got them thinking" about their behaviour, which is what the intervention had been designed to do:

I think what was quite powerful is that when I spoke to the pharmacist then it got me thinking about actually the things I have done at university, and how I was different now, and how l'd changed a little bit and how my drinking at university was clearly to excess, and now how I wanted to regulate and stop that. 120.

Others went further and said they had "cut down" their drinking:

I know that drinking is bad and drinking to excess is bad and l've cut down on my drinking a lot since I first went to the pharmacy and took part in the study. I don't drink half as much as I used to. 116

What it did do ... I didn't drink for the whole of January for various reasons, because I just wanted to see if I could do it, and I did. But also for me who is someone that has given up smoking and continues to battle with that on a social level, it really highlighted to me that in my head smoking and drinking go together, so the less I do it the better. 122

As with the intervention group, around half of the control group said that that taking part had not changed their thinking or their drinking. The others said that talking to the pharmacist during assessment or reading the leaflet had made a difference to how they thought about their drinking, and in a few cases they had made a change to their behaviour: 
I've eased up on it, instead of drinking three cans of beers, just drinking probably two. C11

\section{DISCUSSION}

Can research participation effects explain a null finding? In the case of this community pharmacist brief intervention trial, it appears that they may do. Taking part in the trial itself provided a stimulus to thinking about drinking. Being screened with the AUDIT was potent for some, as this formed an important part of motivation for enrolment. Such impacts are interestingly in line with the original conception of the AUDIT as a platform for consideration of change (Babor et al., 2001). In this study the AUDIT was administered by practitioners in interviews with both randomised groups, as routinely occurs in brief intervention trials. Discussions with pharmacists and other trial procedures implemented throughout the course of the visit to the pharmacy appeared to help some people think about their drinking to an extent that was not obviously different for the intervention and control groups; the dedicated discussion time did not obviously add much in this recalled experience. It is important to be clear about the observational rather than randomised nature of this finding, and also that it is an inadvertent artefact of trial design, and thus does not measure any intervention effect per se. This finding also reflects quantitative data on recall; when asked at trial follow-up whether participants recalled having a discussion with the pharmacist about their drinking following the AUDIT questions, $61 \%$ of control group participants reported doing so, as compared to 77\% of intervention group participants (Dhital et al., 2015). Other well conducted brief intervention trials with null findings also report similar data on recall (Saitz et al., 2007). Participants appeared to regard the pharmacists in ways similar to their GPs, which suggests potential for effective interventions to promote or protect health and wellbeing. These qualitative findings do indeed help to explain the null finding in the trial (Dhital et al., 2015). There is an important need to investigate what participants in brief intervention trials understand, both about the nature of the intervention, and also their experience of participating in the study.

Empathic pharmacists appear to have exerted some influence on the control group that was not intended by the trial design, and any impact of the empathic understanding pharmacist in the 10 minute discussion may have been obscured by the deployment of this manner in alcohol focused exchanges in other parts of the research process. If so, this makes it possible that the intervention effect has been under-estimated, as contamination has occurred. The pharmacists' communication skills were deployed throughout the trial; in the recruitment process, the administration of the AUDIT and the implementation of the consent process, rather than just in the brief intervention discussion. It should be emphasised, however, that mean reductions in drinking reported were very modest (Dhital et al. 2015) and such reductions may be explained by other factors (McCambridge et 
al. 2013). On the basis of this study, we thus hypothesise that brief intervention trials are more likely to produce null findings when clinicians are involved in recruitment and screening.

This is a small study based on a brief interview requiring recall, so the depth of these data is limited. To explore the issues under investigation in greater depth, we recommend future studies with greater resources involving longer (60 - 90 minute) face-to-face interviewing. Although participants were randomly selected, it is difficult to appreciate the implications of any missing data from those who declined to participate in this process study. The transferability of these findings needs to be carefully considered. Although the interviewer was not blind to allocation and this may have affected how the interviews were conducted, the data do not suggest this was a problem. Process studies are not widely implemented in brief intervention trials and these findings suggest that they should be, as they also identify other data relevant to the null finding, such as trial protocol departures.

This study also reveals how widely views about the nature of alcohol problems shape participant engagement with interventions designed to encourage people to reflect on their drinking and to change it. Because most participants had no sense that their drinking was problematic, according to their own preconceived ideas of what this meant, they discounted efforts to help them think about their drinking as not being relevant to them. There is missing proof of concept and evidence of acceptability among this pharmacy study population whose drinking is hazardous or risky, rather than harmful or problematic. It is unclear how widely this disjuncture between the intervention aims which are largely to do with prevention of future harm, and participants' understanding of their needs may be in the existing literature.

This study offers numerous directions for research on null findings in the brief alcohol intervention literature, and indeed in behavioural intervention trials more broadly. These findings, though lacking depth, are in line with previous consideration of the implications of research participation effects for the design of trials, indicating the need to consider in advance potential artefacts in order to avoid them (McCambridge et al. 2014b). We recommend that process studies nested in trials examine research participation effects prospectively along with acceptability issues in other settings because they have potentially major implications for brief, and indeed all behavioural, intervention studies. 


\section{References}

Babor, T.F., Higgins-Biddle, J.C., Saunders, J.B., Monteiro, M.G., 2001. AUDIT - Alcohol Use Disorders Identification Test: Guidelines for Use in Primary Care. Geneva: World Health Organization.

Clifford, P.R., Maisto, S.A., 2000. Subject reactivity effects and alcohol treatment outcome research. J. Stud. Alcohol. 61(6),787-93.

Dhital, R., Norman, I., Whittlesea, C., McCambridge, J., 2013. Effectiveness of alcohol brief intervention delivered by community pharmacists: study protocol of a two-arm randomised controlled trial. BMC public health. 13,152.

Dhital, R., Norman, I., Whittlesea, C., Murrells, T., McCambridge, J. 2015. The effectiveness of brief alcohol interventions delivered by community pharmacists: randomised controlled trial. Addiction. 110(10):1586-94.

Gallen M., 1974. Toward an understanding of follow-up research with alcoholics. Psychol. Rep. 34(3), 877-8.

Gaume, J., McCambridge, J., Bertholet, N., Daeppen, J.B. 2014. Mechanisms of action of brief alcohol interventions remain largely unknown - a narrative review. Frontiers in psychiatry. 5,108.

Groves, R.M., Kahn, R.L. (1979) Surveys by telephone: A national comparison with personal interviews. New York, NY: Academic Press.

Heather N., 1989. Psychology and brief interventions. British Journal of Addiction. 84(4), 357-70.

Irvine, A. 2011. Duration, dominance and depth in telephone and face-to-face interviews: a comparative evaluation. International Journal of Qualitative Methods. 10(3), 202-220.

Jenkins, R.J., McAlaney, J., McCambridge, J. 2009. Change over time in alcohol consumption in control groups in brief intervention studies: systematic review and meta-regression study. Drug Alcohol Depend. 100(1-2), 107-14.

McCambridge, J., 2009. Research assessments: instruments of bias and brief interventions of the future? Addiction. 104(8), 1311-2.

McCambridge, J. 2011. Fifty years of brief intervention effectiveness trials for heavy drinkers. Drug \& Alcohol Rev. 30(6), 567-8.

McCambridge, J., 2013. Brief intervention content matters. Drug \& Alcohol Rev. 32, 339-41.

McCambridge, J., Kypri, K., 2011. Can simply answering research questions change behaviour? Systematic review and meta analyses of brief alcohol intervention trials. PLoS One. 6(10), e23748.

McCambridge. J., Butor-Bhavsar, K., Witton, J., Elbourne, D., 2011. Can research assessments themselves cause bias in behaviour change trials? A systematic review of evidence from Solomon 4group studies. PLoS ONE. 6(10), e25223.

McCambridge, J., K. Kypri and P. McElduff (2013). Regression to the mean and alcohol consumption: A cohort study exploring implications for the interpretation of change in control groups in brief intervention trials. Drug \& Alcohol Dep. 135: 156-159, 
McCambridge, J., Witton, J., Elbourne, D., 2014a. Systematic review of the Hawthorne effect: New concepts are needed to study research participation effects. J. Clin. Epidemiol. 67(3), 267-77.

McCambridge, J., Kypri, K., Elbourne, D., 2014b. In randomisation we trust? There are overlooked problems in experimenting with people in behavioural intervention trials. J. Clin. Epidemiol. 67(3), 247-53.

McCambridge, J., Kypri, K., Elbourne, D., 2014c. Research participation effects: A skeleton in the methodological cupboard J. Clin. Epidemiol. 67, 845-9.

McCambridge, J., A. Sorhaindo, A. Quirk and K. Nanchahal (2014). Patient preferences and performance bias in a weight loss trial with a usual care arm. Patient Educ. Couns. 95(2): 243-247.

Miller, W.R., Rollnick, S., 2012 Motivational Interviewing, Third Edition: Helping People Change. London: The Guilford Press.

Oakley, A., Strange, V., Bonell, C., Allen, E., Stephenson, J., 2006. Process evaluation in randomised controlled trials of complex interventions. BMJ. 332(7538), 413-6.

Ogborne, A.C., Annis, H.M., 1988. The reactive effects of follow-up assessment procedures: an experimental study. Addict. Behav. 13(2), 123-9.

Ritchie, J., Spencer, L., 2002. Qualitative data analysis for applied policy research. In M. Huberman, M.B. Miles (eds). The Qualitative Researcher's Companion. London: Sage.

Saitz, R., Palfai, T.P., Cheng, D.M., Horton, N.J., Freedner, N., Dukes, K., et al., 2007. Brief intervention for medical inpatients with unhealthy alcohol use: a randomized, controlled trial. Ann. Intern. Med. 146(3), 167-76.

Sarantakos, S. (1998) Social Research ( $2^{\text {nd }}$ ed.) South Melbourne, Australia: MacMillan.

Scott, C., Walker, J., White, P., Lewith, G., 2011. Forging convictions: the effects of active participation in a clinical trial. Soc. Sci. Med. 72(12), 2041-8.

Sobell, L.C., Sobell, M.B., 1981. Frequent follow-up as data gathering and continued care with alcoholics. Int. J. Addict. 16(6), 1077-86. 
Table 1

\begin{tabular}{llll}
\hline Characteristics & $\begin{array}{l}\text { Intervention group } \\
(\boldsymbol{n}=\mathbf{1 2})\end{array}$ & $\begin{array}{l}\text { Control group } \\
(\boldsymbol{n}=\mathbf{1 2})\end{array}$ & $\begin{array}{l}\text { Total } \\
(\boldsymbol{n}=\mathbf{2 4}\end{array}$ \\
\hline Age years: mean $(S D)$, range & $36.0(14.2), 22-69$ & $41.1(17.9), 19-67$ & $38.5(16.0), 19-69$
\end{tabular}

Gender:

Female

$7(58.3)$

$4(33.3)$

11 (45.8)

Male

5 (41.7)

$8(66.7)$

13 (54.2)

Ethnicity:

White: British

$10(83.3)$

$6(50.0)$

$16(66.7)$

Other

2 (16.7)

$6(50.0)$

$8(33.3)$

\section{Continuing education}

after age 16:

Yes

$8(66.7)$

10 (83.3)

$18(75.0)$

No

$4(33.3)$

2 (16.7)

$6(25.0)$ 